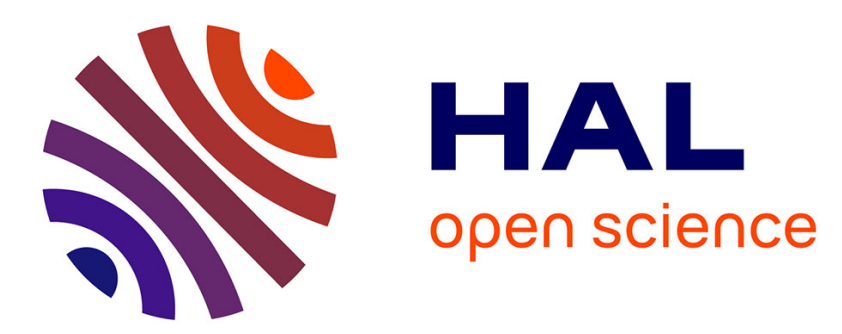

\title{
Flying Ad Hoc Network for Emergency Applications connected to a Fog System
}

Dan Radu, Adrian Cretu, Benoît Parrein, Jiazi Yi, Camelia Avram, Adina Astilean

\section{- To cite this version:}

Dan Radu, Adrian Cretu, Benoît Parrein, Jiazi Yi, Camelia Avram, et al.. Flying Ad Hoc Network for Emergency Applications connected to a Fog System. Emerging Internet, Data \& Web Technologies (EIDWT) 2018, Mar 2018, Tirana, Albania. pp.675-686, 10.1007/978-3-319-75928-9_60 . hal-01763827

\section{HAL Id: hal-01763827 \\ https://hal.science/hal-01763827}

Submitted on 11 Apr 2018

HAL is a multi-disciplinary open access archive for the deposit and dissemination of scientific research documents, whether they are published or not. The documents may come from teaching and research institutions in France or abroad, or from public or private research centers.
L'archive ouverte pluridisciplinaire HAL, est destinée au dépôt et à la diffusion de documents scientifiques de niveau recherche, publiés ou non, émanant des établissements d'enseignement et de recherche français ou étrangers, des laboratoires publics ou privés. 


\title{
Flying Ad Hoc Network for Emergency Applications connected to a Fog System
}

\author{
Dan Radu, Adrian Cretu, Benoît Parrein, Jiazi Yi, Camelia Avram, Adina Aştilean
}

\begin{abstract}
The main objective of this paper is to improve the efficiency of vegetation fire emergency interventions by using MP-OLSR routing protocol for data transmission in Flying Ad Hoc NETwork (FANET) applications. The presented conceptual system design could potentially increase the rescuing chances of people caught up in natural disaster environments, the final goal being to provide public safety services to interested parties. The proposed system architecture model relies on emerging technologies (Internet of Things \& Fog, Smart Cities, Mobile Ad Hoc Networks) and actual concepts available in the scientific literature. The two main components of the system consist in a FANET, capable of collecting fire detection data from GPS and video enabled drones, and a Fog/Edge node that allows data collection and analysis, but also provides public safety services for interested parties. The sensing nodes forward data packets through multiple mobile hops until they reach the central management system. A proof of concept based on MP-OLSR routing protocol for efficient data transmission in FANET scenarios and possible public safety rescuing services is given.
\end{abstract}

Dan Radu

Technical University of Cluj-Napoca e-mail: dan.radu@aut.utcluj.ro

Adrian Cretu

Technical University of Cluj-Napoca e-mail: adriancr.ro@gmail.com

Benoit Parrein

LS2N, Polytech'Nantes e-mail: benoit.parrein@polytech.univ-nantes.fr

Jiazi Yi

Laboratoire d'Informatique, Ecole Polytechnique, France e-mail: yi@lix.polytechnique.fr

Camelia Avram

Technical University of Cluj-Napoca e-mail: camelia.avram@aut.utcluj.ro

Adina Astilean

Technical University of Cluj-Napoca e-mail: adina.astilean@aut.utcluj.ro 


\section{Introduction}

The main objective of this paper is to introduce MP-OLSR routing protocol, that already proved to be efficient in MANET and VANET scenarios Yi et al (2011a), Radu et al (2012), into FANET applications. Furthermore, as a proof of concept, this work presents a promising smart system architecture that can improve the saving chances of people caught in wildfires by providing real-time rescuing services and a temporary communication infrastructure. The proposed system could locate the wildfire and track the dynamics of its boundaries by deploying a FANET composed of GPS and video enabled drones to monitor the target areas. The video data collected from the FANET is sent to a central management system that processes the information, localizes the wildfire and provides rescuing services to the people (fire fighters) trapped inside wildfires. The data transmission QoS (Quality of Service) for data transmission in the proposed FANET network scenario is provided to prove the efficiency of MP-OLSR, a multipath routing protocol based on OLSR, in this types of applications.

Wildfires are unplanned events that usually occur in natural areas (forests, prairies) but they could also reach urban areas (buildings, homes). Many such events occured the last years (e.g. Portugal and Spain 2017, Australia 2011). The forrest fire in the north of Portugal and Spain killed more than 60 people. During the Kimberley Ultra marathon held in Australia in 2011 multiple persons were trapped in a bush fire that started during a sports competition.

The rest of this paper is structured as folows. Section 2 presents the related works in the research field. Section 3 introduces the proposed system design. Section 4 shows and discusses the QoS performance evaluation results. Finally, Section 5 concludes the paper.

\section{Related works}

Currently there is a well-known and increasing interest for providing Public Safety services in case of emergency/disaster situations. The US Geospatial Multi Agency Coordination ${ }^{1}$ provides a web service that displays fire dynamics on a map by using data gathered from different sources (GPS, infrared imagery from satellites). A new method for detecting forest fires based on the color index was proposed in Cruz et al (2016). Authors suggest the benefits of a video surveillance system installed on drones. Another system, composed of unmanned aerial vehicles, used for dynamic wildfire tracking is discussed in Pham et al (2017).

This section presents the state of the art of the concepts and technologies used for the proposed system design, current trends, applications and open issues.

\footnotetext{
${ }^{1}$ https://www.geomac.gov
} 


\subsection{Internet of Things and Fog Computing}

Internet of Things (IoT), Fog Computing, Smart Cities, Unmanned Aerial Vehicle Networks, Mobile Ad Hoc Networks, Image Processing Techniques and Algorithms, and Web Services are only some of the most promising actual, emerging technologies. These all share a great potential to be used together in a large variety of practical applications that could improve, sustain and support peoples life.

There are many comprehensive surveys in the literature that analyse the challenges of IoT and provide insights over the enabling technologies, protocols and possible applications Al-Fuqaha et al (2015). In the near future, traditional cloud computing based architectures will not be able to sustain the IoT exponential growth leading to latency, bandwidth and inconsistent network challenges. Fog computing could unlock the potential of such IoT systems.

Fog computing refers to a computing infrastructure that allows data, computational and business logic resources and storage to be distributed between the data source and the cloud services in the most efficient way. The architecture could have a great impact in the emerging IoT context, in which billions of devices will transmit data to remote servers, because its main purpose is to extend cloud infrastructure by bringing the advantages of the cloud closer to the edge of the network where the data is collected and pre-processed. In other words, fog computing is a paradigm that aims to efficiently distribute computational and networking resources between the IoT devices and the cloud by:

- allowing resources and services to be located closer or anywhere in between the cloud and the IoT devices;

- supporting and delivering services to users, possibly in an offline mode when the network is partitioned by example;

- extending the connectivity between devices and the cloud across multiple protocol layers.

In the near future, traditional cloud computing based architectures will not be able to sustain the IoT exponential growth leading to latency, bandwidth and inconsistent network challenges. Fog computing could unlock the potential of such IoT systems.

Currently the use cases and the challenges of the edge computing paradigm are discussed in various scientific works Lin et al (2017), Al-Fuqaha et al (2015), Ang et al (2017). Some of the well known application domains are: energy, logistics, transportation, healthcare, industrial automation, education and emergency services in case of natural or man made disasters. Some of the challenging Fog computing research topics are: crowd based network measurement and interference, client side network control and configuration, over the top content management, distributed data centers and local storage/computing, physical layer resource pooling among clients, Fog architecture for IoT, edge analytics sensing, stream mining and augmented reality, security and privacy.

There are numerous studies that connect video cameras to Fog \& IoT applications. The authors of Shi et al (2016) discuss a couple of practical usages for Fog 
computing: cloud offloading, video analytics, smart home and city, and collaborative edge. Also, some of the research concepts and opportunities are introduced: computing stream, naming schemes, data abstraction, service management, privacy and security, and optimization metrics. Authors of Shi and Dustdar (2016) present a practical use case in which video cameras are deployed in public areas or on vehicles and they could be used to identify a missing persons image. In this case, the data processing and identification could be done at the edge without the need of uploading all the video sources to the cloud. A method that distributes the computing workload between the edge nodes and the cloud was introduced Zhang et al (2016). Authors try to optimize data transmission and ultimately increase the life of edge devices such as video cameras.

Fog computing could be the solution to some of the most challenging problems that arise in the Public Safety domain. Based on the most recent research studies and previous works concerning public safety Radu et al (2012), Yi et al (2011b) it can be stated that real time image \& video analysis at the edge of a FANET network could be successfully implemented in the public safety domain, more specifically for fire detection and for rescuing emergency services provisioning.

One of the most important advantages of Fog computing is the distributed architecture that promises better Quality of Experience and Quality of Service in terms of response, network delays and fault tolerance. This aspect is crucial in many Public Safety applications where data processing should be done at the edge of the system and the response times have hard real-time constraints.

\subsection{Flying Ad Hoc Networks}

Unmanned Aerial Vehicles (UAV's, commonly known as drones) become more and more present in our daily lifes through their ease of deployment in areas of interest. The high mobility of the drones, with their enhanced hardware and software capabilities, makes them suitable for a large variety of applications including transportation, farming and disaster management services. FANET's are considered as a sub type of Mobile Ad Hoc Networks networks that have a greater degree of mobility and usually the distance between nodes is greater as stated in Bekmezci et al (2013).

A practical FANET testbed, build on top of Raspberry $\mathrm{Pi}^{\circledR}{ }^{\circledR}$, that uses two WiFi connections on each drone (one for ad hoc network forwarding and the other for broadcasted control instructions is described in Bekmezci et al (2015). Another FANET implementation that consists of quadcopters for disaster assistance, search and rescue and aerial monitoring as well as the design challenges are presented in Yanmaz et al (2018) 


\subsection{Routing protocols}

OLSR (Optimized Link State Routing) protocol proposed in Jacquet et al (2001) is an optimization of link state protocol. This single path routing approach presents the advantage of having shortest path routes immediately available when needed (proactive routing). OLSR protocol has low latency and performs best in large and dense networks.

In Haerri et al (2006) OLSR and AODV are tested against node density and data traffic rate. Results show that OLSR outperforms AODV in VANETs, providing smaller overhead, end-to-end delay and route lengths. Furthermore there are extensive studies in the literature regarding packets routing in FANET's. Authors of Oubbati et al (2017) give a classification and taxonomy of existing protocols as well as a complete description of the routing mechanisms for each considered protocol. An example of a FANET specific routing protocol is an adaptation of OLSR protocol that uses GPS information and computes routes based on the direction and relative speed between the UAV's is proposed in Rosati et al (2016).

In this paper authors use MP-OLSR (Multiple Paths OLSR) routing protocol based on OLSR proposed in Yi et al (2011a), that allows packet forwarding in FANET and MANET networks through spatially separated multiple paths. MPOLSR exploits simultaneously all the available and valuable multiple paths between a source and a destination to balance traffic load and to reduce congestion and packet loss. Also it provides a flexible degree of spatial separation between the multiple paths by penalizing edges of the previous paths in an original Dijkstra algorithm execution.

Based on the above considerations, a system architecture that can improve the saving chances of people caught in wildfires by providing real-time rescuing services and a temporary communication infrastructure is proposed.

\section{System Design}

One of the main objectives of this work is to design and develop a smart system architecture, based on FANET networks, which integrates with the numerous emergent applications offered by the Internet of Things, that is:

- extensible: the system architecture should allow any new modules to be easily plugged in;

- reliable: the system should support different levels of priority and quality of service for the modules that will be plugged in. For example, the public safety and emergency services that usually have real-time hard constraints should have a higher priority than other services that are not critical;

- scalable: the architecture should support the connection of additional new Fog components, features and high node density scenarios; 
- resilient: the system will be able to provide and maintain an acceptable level of service whenever there are any faults to the normal operation.

The overview of the proposed model, in the context of Internet of Things \& Fog Computing, is given in Figure 1.

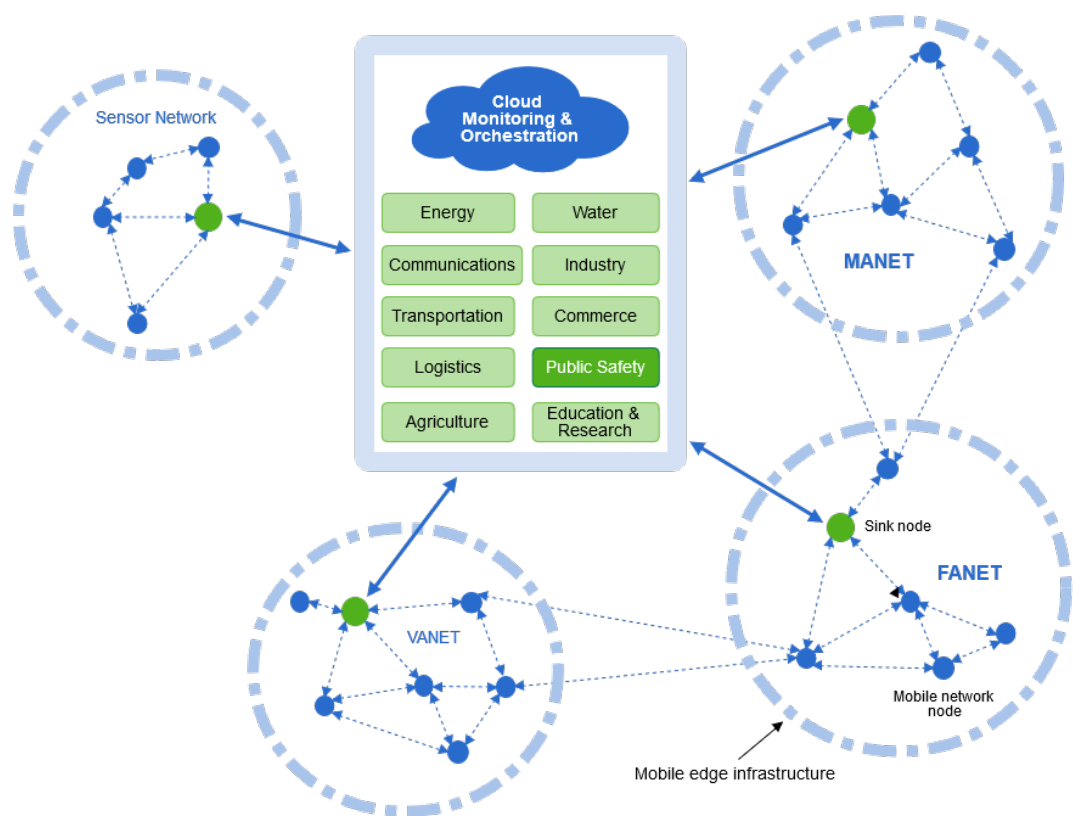

Fig. 1 System overview

The system could locate the wildfire and track the dynamics of its boundaries by deploying a flying ad hoc network composed of GPS and video enabled drones to monitor the target areas. The fire identification data collected from the FANET is sent to a central management system that processes the data, localizes the wildfire and provides rescuing services to the people (fire fighters) trapped inside the wildfires. The proposed system intends to support and improve emergency intervention services by integrating, based on the real-time data collected from the Fog network, multiple practical services and modules such as:

- affected area surveillance;

- establishing the communication network between the disaster survivors and rescue teams;

- person in danger identification and broadcasting of urgent notifications;

- supporting the mobility of the first responders through escape directions;

- rescuing vehicle navigation.

Our FEA (FANET Emergency Application) network topology is presented in Figure 2 and it is composed of three main components: 
- A MANET of mobile users phones;

- FANET - video and GPS equipped drones that also provide sufficient computational power capabilities for fire pattern recognition;

- Fog infrastructure that supports FANET data collection at the sink node located at the edge of the network. This provides data storage, computational power and supports different communication technologies for the interconnection with other edge systems.

This last component can be done through an object store as proposed in Confais et al (2017b) where a traditional Bittorrent P2P network can be used for storage purpose. Combined with a Scale-out NAS as in Confais et al (2017a), the Fog system avoids costly metadata management (even in local accesses) and computing capacity thanks to an intensive I/O distributed file system. Moreover, the global Fog system allows to work on a disconnected mode in case of network partitionning with the backbone.

FEA uses a FANET network, to collect fire identification data from drones (GPS and video enabled), and a MANET network composed of users smartphones. Sensing nodes periodically transmit data to the central management system where the fire dynamics is determined for monitoring purposes. If a fire has been detected by a sensing drone, based on the dynamics of the fire, rescuing information will be computed and broadcasted back into the FANET and MANET so that the people trapped in the fire to be able to receive the safety information on their smartphones in real-time. We make the folowing assumptions, that will be taken into account for the simulation scenario modelling, regarding the FEA message forwarding:

- when fire is detected by sensing drones they start to periodically forward data packets with the information regarding fire dynamics over multiple hops in the mesh network towards the sink node;

- the central management system processes the fire detection data received from FANET nodes and computes the fire dynamics using the GPS coordinates that are included in the received data messages;

- the central management system sends back into the mobile network (FANET and MANET) rescuing information that will be received by people in danger on their smartphones.

In FEA system FANET nodes are responsible for: fire identification based on video recording, forwarding the processed information (alongside with GPS coordinates) towards the collector node and rescuing information forwarding to the MANET nodes. The proposed network architecture could also serve as a temporary communication infrastructure between rescuing teams and people in danger.

One of the many advantages of FEA is the ease of deployment, all the technologies and components of the system are widely available, inexpensive and easy to provide. Also the Quality of Service in the FANET network, which is essential in emergency services where delays and packet delivery rations are very important, is enhanced by using MP-OLSR routing protocol that chooses the best multiple paths available between source and destination. 


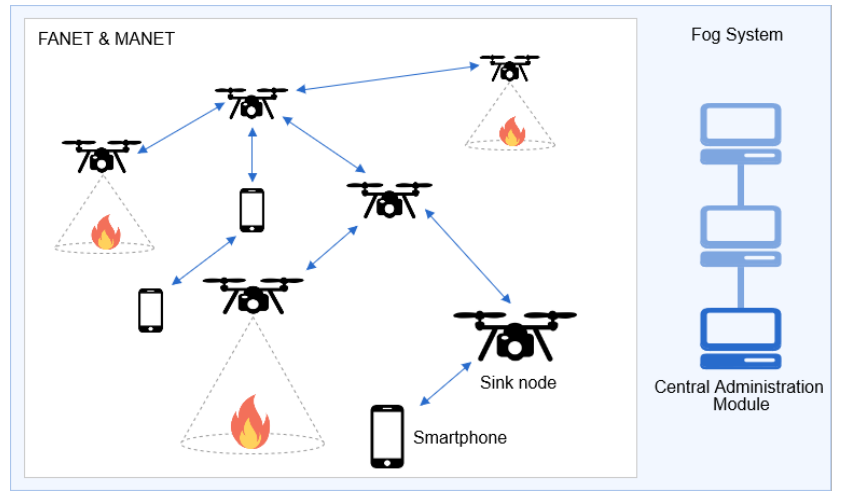

Fig. 2 Emergency system architecture

\section{System Evaluation}

The simulations are performed to evaluate MP-OLSR in the proposed FANET scenario. This section is organized as follows. The simulation environment configuration and scenario assumptions are given in Section 4.1 and then the Quality of Service performance are compared between OLSR and MP-OLSR in Section 4.2

\subsection{Simulation Scenario}

For the simulations we designed a 81 nodes FANET \& MANET hybrid topology placed in a 1480 square meters grid topology. The Random Waypoint Model mobility pattern was used for different maximal speeds suitable for the high mobility of drones: $1-15 \mathrm{~m} / \mathrm{s}(3.6-54 \mathrm{~km} / \mathrm{h})$. We make the assumption that only a subset of nodes (possibly the ones that detect fire or the smart phones of people in danger) need to communicate with the Fog edge node through the mesh network so the data traffic is provided by 4 Constant Bit Rate (CBR) sources. Qualnet 5 was used as a discrete event network simulator. The detailed parameters for the Qualnet network scenario and routing protocols configuration parameters are listed in Table 1. The terrain altitude profile is shown in Figure 3.

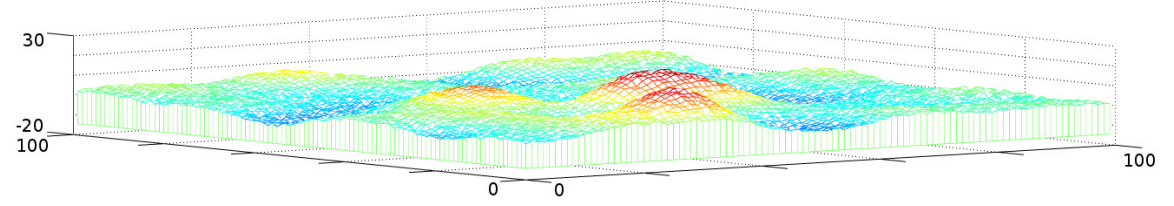

Fig. 3 Qualnet altitude profile pattern for $100 \mathrm{~m}^{2}$ 
Table 1 Simulation parameters.

\begin{tabular}{llll}
\hline Simulation Parameter & Value & Routing Parameter & Value \\
\hline Simulator & Qualnet 5 & TC Interval & $5 \mathrm{~s}$ \\
Routing protocols & OLSRv2 and MP-OLSR & HELLO Interval & $2 \mathrm{~s}$ \\
Area & $1480 \times 1480 \times 34.85 \mathrm{~m}^{3}$ & Refresh Timeout Interval & $2 \mathrm{~s}$ \\
Number of nodes & 81 & Neighbor hold time & $6 \mathrm{~s}$ \\
Initial nodes placement & Grid & Topology hold time & $15 \mathrm{~s}$ \\
Mobility model & Random Waypoint & Duplicate hold time & $30 \mathrm{~s}$ \\
Speeds & $1-15 \mathrm{~m} / \mathrm{s}$ & Link Layer Notification & Yes \\
Number of seeds & 10 & No. of path in MP-OLSR & 3 \\
Transport protocol & UDP & & \\
IP & IPv4 & & \\
IP fragmentation unit & 2048 bytes & & \\
Physical layer model & PHY 802.11b & & \\
Link layer data rate & $11 \mathrm{Mbits} / \mathrm{s}$ & & \\
Number of CBR sources & 4 & & \\
Sim duration & $100 \mathrm{~s}$ & & \\
CBR start-end & $15-95 \mathrm{~s}$ & & \\
Transmission interval & $0.05 \mathrm{~s}$ & & \\
Application packet size & 512 bytes & & \\
\hline
\end{tabular}

\subsection{Simulation Results}

For each routing protocol a number of 80 simulations were executed (10 different seeds/speed ranges). To compare the performances of the protocols, the following metrics are used:

- Packet delivery ratio (PDR): the ratio of the data packets successfully delivered at all destinations.

- Average end-to-end delay: averaged over all received data packets from sources to destinations as depicted in Schulzrinne et al (1996).

- Jitter: average jitter is computed as the variation in the time between packets received at the destination caused by network congestions and topology changes.

Figures 4, 5 and 6 show the QoS performance of MP-OLSR and OLSR in terms of PDR, end-to-end delay and Jitter results with standard deviation for each point. From the obtained results it can be seen that PDR decreases slightly with the mobility as expected. For the proposed FANET scenario MP-OLSR delivers an average of $10 \%$ higher PDR than OLSR protocol.As expected, when the speed increases to values closer to the high mobility of FANET scenarios the links become more unstable so OLSR performance decreases while MP-OLSR provides a much better overall delivery ratio than OLSR (around 9\% in average at higher speeds). MP-OLSR also performs much better than OLSR in terms of end-to-end delay and Jitter. The delay of OLSR is around 2 times higher at the highest speed while Jitter is $50 \%$ higher. This aspect is very important for the proposed emergency application where the re- 
sponse time must be provided as quickly as possible. Furthermore, the MP-OLSR standard deviation for all the results is smaller than for OLSR.

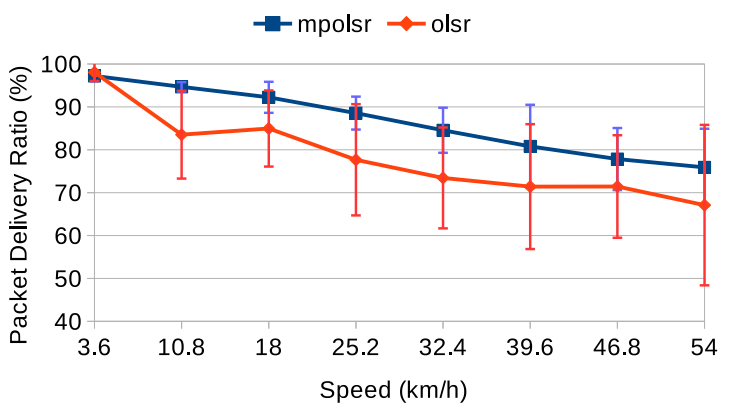

Fig. 4 Delivery ratio

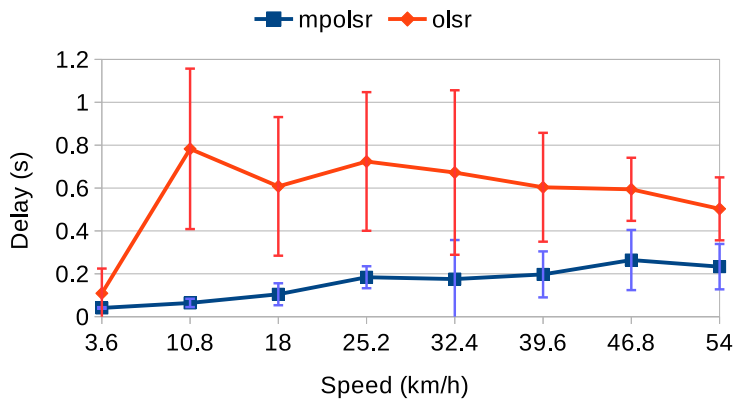

Fig. 5 End-to-end delay

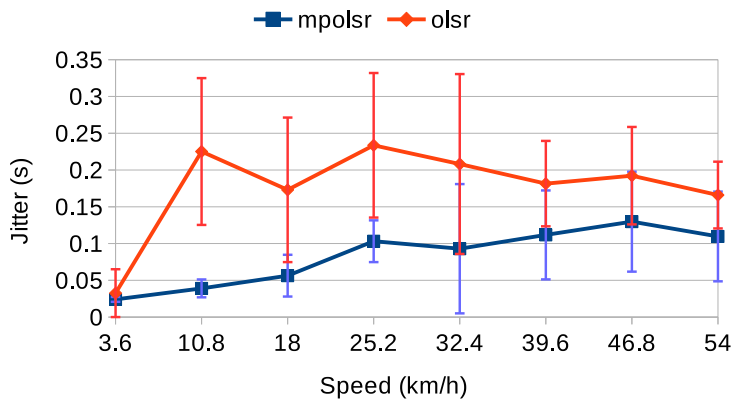

Fig. 6 Jitter 


\section{Conclusion and Future Work}

We described FEA system as a possible emergency application for MP-OLSR routing protocol which uses a FANET network to collect fire dynamics data from drones and through a central management system it provides safety instructions back to the people in danger. The performance evaluation results show that MP-OLSR is suitable for FANET scenarios, most specifically emergency applications, where the mobility is high and response times have hard real-time constraints.

The folowing are some of our future works: system deployment on a real testbed, analysis of the cooperation between MANET \& FANET, data analysis based on thermal cameras.

\section{References}

Al-Fuqaha A, Guizani M, Mohammadi M, Aledhari M, Ayyash M (2015) Internet of things: A survey on enabling technologies, protocols, and applications. IEEE Communications Surveys Tutorials 17(4):2347-2376, DOI 10.1109/COMST.2015.2444095

Ang LM, Seng KP, Zungeru AM, Ijemaru GK (2017) Big sensor data systems for smart cities. IEEE Internet of Things Journal 4(5):1259-1271, DOI 10.1109/JIOT.2017.2695535

Bekmezci I, Sahingoz OK, amil Temel (2013) Flying ad-hoc networks (fanets): A survey. Ad Hoc Networks 11(3):1254 - 1270, DOI https://doi.org/10.1016/j.adhoc.2012.12.004, URL http://www.sciencedirect.com/science/article/pii/S1570870512002193

Bekmezci I, Sen I, Erkalkan E (2015) Flying ad hoc networks (fanet) test bed implementation. In: 2015 7th International Conference on Recent Advances in Space Technologies (RAST), pp 665-668, DOI 10.1109/RAST.2015.7208426

Confais B, Lèbre A, Parrein B (2017a) An Object Store Service for a Fog/Edge Computing Infrastructure based on IPFS and Scale-out NAS. In: 1st IEEE International Conference on Fog and Edge Computing - ICFEC'2017, Madrid, Spain, URL https://hal.archives-ouvertes.fr/hal-01483702

Confais B, Lèbre A, Parrein B (2017b) Performance Analysis of Object Store Systems in a Fog and Edge Computing Infrastructure. Transactions on LargeScale Data- and Knowledge-Centered Systems DOI 10.1007/978-3-662-556962_2, URL https://hal.archives-ouvertes.fr/hal-01587459

Cruz H, Eckert M, Meneses JM, Martínez J (2016) Efficient forest fire detection index for application in unmanned aerial systems (uass). Sensors 16(6):893

Haerri J, Filali F, Bonnet C (2006) Performance comparison of aodv and olsr in vanets urban environments under realistic mobility patterns. In: Med-Hoc-Net 2006, 5th IFIP Mediterranean Ad-Hoc Networking Workshop, June 14-17, Lipari, ITALY 
Jacquet P, Muhlethaler P, Clausen T, Laouiti A, Qayyum A, Viennot L (2001) Optimized link state routing protocol for ad hoc networks. In: Multi Topic Conference, 2001. IEEE INMIC 2001. Technology for the 21st Century. Proceedings. IEEE International, pp 62-68

Lin J, Yu W, Zhang N, Yang X, Zhang H, Zhao W (2017) A survey on internet of things: Architecture, enabling technologies, security and privacy, and applications. IEEE Internet of Things Journal 4(5):1125-1142, DOI 10.1109/JIOT.2017.2683200

Oubbati OS, Lakas A, Zhou F, Gne M, Yagoubi MB (2017) A survey on position-based routing protocols for flying ad hoc networks (fanets). Vehicular Communications DOI https://doi.org/10.1016/j.vehcom.2017.10.003, URL http://www.sciencedirect.com/science/article/pii/S2214209617300529

Pham HX, La HM, Feil-Seifer D, Deans M (2017) A distributed control framework for a team of unmanned aerial vehicles for dynamic wildfire tracking. CoRR abs/1704.02630, URL http://arxiv.org/abs/1704.02630, 1704.02630

Radu D, Avram C, Atilean A, Parrein B, Yi J (2012) Acoustic noise pollution monitoring in an urban environment using a vanet network. In: Proceedings of 2012 IEEE International Conference on Automation, Quality and Testing, Robotics, pp 244-248, DOI 10.1109/AQTR.2012.6237711

Rosati S, Kruelecki K, Heitz G, Floreano D, Rimoldi B (2016) Dynamic routing for flying ad hoc networks. IEEE Transactions on Vehicular Technology 65(3):16901700, DOI 10.1109/TVT.2015.2414819

Schulzrinne H, Casner S, Frederick R, Jacobson V (1996) Rfc 1889: Rtp: A transport protocol for real-time applications. Network Working Group Memorandum, http: //www.faqs.org/rfcs/rfc1889.html

Shi W, Dustdar S (2016) The promise of edge computing. Computer 49(5):78-81, DOI doi.ieeecomputersociety.org/10.1109/MC.2016.145

Shi W, Cao J, Zhang Q, Li Y, Xu L (2016) Edge computing: Vision and challenges. IEEE Internet of Things Journal 3(5):637-646, DOI 10.1109/jiot.2016.2579198, URL http://dx.doi.org/10.1109/jiot.2016.2579198

Yanmaz E, Yahyanejad S, Rinner B, Hellwagner H, Bettstetter C (2018) Drone networks: Communications, coordination, and sensing. Ad Hoc Networks 68(Supplement C):1 - 15, DOI https://doi.org/10.1016/j.adhoc.2017.09.001, URL http://www.sciencedirect.com/science/article/pii/S1570870517301671, advances in Wireless Communication and Networking for Cooperating Autonomous Systems

Yi J, Adnane A, David S, Parrein B (2011a) Multipath optimized link state routing for mobile ad hoc networks. Ad Hoc Networks 9(1):28-47

Yi J, Parrein B, Radu D (2011b) Multipath routing protocol for manet: Application to h.264/sve video content delivery. In: 2011 The 14th International Symposium on Wireless Personal Multimedia Communications (WPMC), pp 1-5

Zhang Q, Yu Z, Shi W, Zhong H (2016) Demo abstract: Evaps: Edge video analysis for public safety. 2016 IEEE/ACM Symposium on Edge Computing (SEC) 00(undefined):121-122, DOI doi.ieeecomputersociety.org/10.1109/SEC.2016.30 